12

\title{
Влияние внешнего продольного электрического поля на трекинг-силу при взаимодействии релятивистского электронного пучка с ионным каналом в режиме ионной фокусировки
}

\author{
(C) Е.К. Колесников, А.С. Мануйлов
}

Санкт-Петербургский государственный университет, 199034 Санкт-Петербург, Россия

e-mail: a.manuylov@spbu.ru, man06@mail.ru

(Поступило в Редакцию 25 января 2017 г.)

Показано, что при наличии продольного внешнего электрического поля трекинг-сила или детрекингсила, возникающие при ускорении или торможении пучка, распространяющегося вдоль предварительно созданного ионного канала в режиме ионной фокусировки, по своей величине могут быть соизмеримы с силой взаимодействия между релятивистским электронным пучком и ионным плазменным каналом.

DOI: $10.21883 /$ JTF.2017.12.45216.2181

\section{Введение}

В последние годы внимание исследователей привлекают вопросы распространения релятивистских электронных пучков (РЭП) вдоль предварительно созданных омических и ионных плазменных каналов в плотных и разреженных газоплазменных средах [1-6]. В частности, в работе [5] исследовался процесс транспортировки РЭП вдоль ионных каналов в режиме ионной фокусировки (ИФ). Режим ИФ может иметь место в сильно разреженном фоновом газе, когда электроны предварительно созданного ионного канала удаляются из области, занятой РЭП, с помощью действия радиальной компоненты электрического поля головной части пучка. В этом случае основная часть РЭП распространяется на фоне неподвижного ионного канала (если длительность пучка меньше характерного времени движения ионов в коллективном электромагнитном поле).

В настоящей работе проводится оценка соотношения трекинг-силы при взаимодействии РЭП и неподвижного ионного канала и детрекинг-силы, которая возникает при торможении РЭП во внешнем продольном электрическом поле, направленном по вектору скорости движения электронов пучка.

\section{Постановка и решение задачи}

Рассмотрим параксиальный моноэнергетический аксиально-симметричный РЭП, распространяющийся в разреженном газе вдоль предварительно созданного плазменного канала. Выберем цилиндрическую систему координат $(r, \theta, z)$ так, чтобы ось $z$ совпадала с осью симметрии канала. Давление фонового газа полагаем таким, что выполнены условия существования режима ИФ, а именно [5]

$$
b_{a v} \gg R_{b} \text {. }
$$

Здесь $b_{a v}$ - характерная длина развития лавинной ионизации окружающего пучок газа, $R_{b}-$ характерный радиус пучка. В этой ситуации плазменные электроны под действием поперечной компоненты электрического поля в головной части РЭП удаляются из плазменного канала без дополнительной ионизации фонового газа.

Далее будем считать, что радиальные зависимости объемных концентраций электронов пучка и ионов плазменного канала имеют гауссовский радиальный профиль, а именно

$$
n_{m}(r)=\frac{N_{m}}{\pi R_{m}^{2}} \exp \left(-\frac{r^{2}}{R_{m}^{2}}\right), \quad m=1,2,
$$

где

$$
N_{1}=N_{b}, \quad N_{2}=N_{i}, \quad R_{1}=R_{b}, \quad R_{2}=R_{i} .
$$

Здесь $N_{b}, N_{i}$ - соответственно погонные концентрации электронов пучка и ионов плазменного канала (концентрации, приходящиеся на единицу длины пучка или ионного канала), $R_{b}, R_{i}-$ характерные радиусы пучка и ионного канала.

В рамках модели „жесткого пучка“ предполагаем, что РЭП смещается в поперечном направлении без деформации его радиального профиля концентрации. В этом случае трекинг-сила (возвращающая сила), действующая на смещенный пучок со стороны неподвижного ионного канала, может быть определена как

$$
\left|F_{x}\right|=|e| \frac{N_{b}}{\pi R_{b}^{2}} \int_{\Omega} d \mathbf{r}_{\perp} \Phi(r, \theta, S) E_{r}^{(i)} \cos \theta,
$$

где $e-$ заряд электрона, $S^{2}=r^{2}+Y^{2}-2 r Y \cos \theta, \mathbf{r}_{\perp}-$ радиус-вектор, проведенный из точки пересечения поперечной плоскости и оси ионного канала в точку наблюдения, $\Omega$ - область в поперечной плоскости, занятая пучково-плазменной системой, $\theta-$ угол между осью $x$, вдоль которой происходит смещение пучка и направлением радиус-вектора $\mathbf{r}_{\perp}-$ амплитуда отклонения центра симметрии пучка относительно оси 
ионного канала, функция $\Phi(r, \theta, S)$ в случае гауссовых радиальных профилей пучка и ионного канала имеет следующий вид соответственно

$$
\Phi(r, \theta, S)=\exp \left(\frac{S^{2}}{R_{b}^{2}}\right),
$$

где $E_{r}^{(i)}$ - радиальная компонента напряженности электростатического поля, созданного ионным каналом в точке наблюдения.

В силу предположения о неподвижности ионной компоненты канала имеем

$$
\frac{1}{r} \frac{d}{d r}\left(r E_{r}^{(i)}\right)=4 \pi|e| n_{i}(r),
$$

с граничным условием $\left.E_{r}^{(i)}\right|_{r=0}=0$.

В случае гауссова радиального профиля ионного канала нетрудно получить

$$
E_{r}^{(i)}(r)=\frac{2 \alpha_{n} I_{b}}{\beta c} \frac{r / R_{i}^{2}}{\left(1+r^{2} / R_{i}^{2}\right)},
$$

где $\alpha_{n}=N_{i} / N_{b}-$ коэффициент зарядовой нейтрализации в режиме ИФ, $I_{b}$ - полный ток пучка, $c$ скорость света, $\beta=v_{z} / c-$ отношение продольной компоненты скорости частиц пучка (который полагается моноскоростным в $z$-направлении) к скорости света.

Для упрощения вычислений рассмотрим следующие безразмерные величины:

$$
r_{i}=\frac{r}{R_{b}}, \quad Y_{1}=\frac{Y}{R_{b}}, \quad \eta=\left(\frac{R_{i}}{R_{b}}\right)^{2} .
$$

Тогда с учетом (5)-(8) имеем соответственно для гауссова случая

$$
\begin{aligned}
F^{(G)}= & F_{0} \int_{0}^{2 \pi} d \theta \cos \theta \int_{0}^{\infty} d r \exp \left[-\left(r_{1}^{2}+Y_{1}^{2}-2 r_{1} Y_{1} \cos \theta\right)\right] \\
& \times\left[1-\exp \left(-r_{1}^{2} / \eta\right)\right]
\end{aligned}
$$

где $F_{0}=2 f_{n} I_{b}^{2} /\left[\pi R_{b}(\beta c)^{2}\right]$ - характерная трекинг-сила, приходящаяся на единицу длины пучка.

При отсутстствии потерь энергии в случае неподвижного ионного канала имеем уравнение движения оси симметрии РЭП

$$
\frac{\partial^{2} Y}{\partial z^{2}}=-F_{1} G(Y, \eta)
$$

где $F_{1}=2 f_{n} I_{b} /\left(\pi R_{b} I_{A} \beta^{2}\right)-$ характерная трекинг-сила, приходящаяся на единицу длины пучка,

$$
\begin{aligned}
G\left(Y_{1}, \eta\right)= & \int_{0}^{2 \pi} d \theta \cos \theta \int_{0}^{\infty} d r_{1} \exp \left[-\left(r_{1}^{2}+Y_{1}^{2}-2 r_{1} Y_{1} \cos \theta\right)\right] \\
& \times\left[1-\exp \left(-r_{1}^{2} / \eta\right)\right]
\end{aligned}
$$

Здесь радиальные профили пучка и ионного канала предполагаются беннетовскими, остальные величины представлены в (8) .

Нетрудно показать, что при наличии энергетических потерь уравнение (10) изменится следующим образом:

$$
\frac{1}{\gamma} \frac{\partial}{\partial z}\left(\gamma \frac{\partial Y}{\partial z}\right)=-F_{1} G(Y, \eta)
$$

где $\gamma$ - лоренц-фактор электронов параксиального пучка.

Из уравнения энергии движения электрона пучка при наличии внешнего продольного электронного поля $E_{z}$ получим

$$
\frac{1}{\gamma} \frac{\partial \gamma}{\partial z}=-\frac{\beta^{2} c\left|E_{z}\right| p}{I_{A}}
$$

Здесь $p-$ косинус угла между векторами $\mathbf{E}_{z}$ и $\mathbf{v}_{z}$. Очевидно, что при совпадении указанных векторов по направлению $\partial \gamma / \partial z<0$ энергия электронов в процессе распространения пучка уменьшается. В противном случае имеем $\partial \gamma / \partial z>0$ и энергия электронов нарастает.

Далее введем обозначение

$$
a=-\frac{\beta^{2} c\left|E_{z}\right| p}{I_{A}}
$$

Тогда из уравнения (12) следует

$$
\frac{\partial^{2} Y}{\partial z^{2}}=-a \frac{\partial Y}{\partial z}-F_{1} G(Y, \eta)
$$

Очевидно, что при $a>0$ первое слагаемое в правой части (15) напоминает слагаемое, связанное с фазовым перемешиванием. Тогда это слагаемое работает на стабилизацию поперечных колебаний оси симметрии РЭП. Это соответствует случаю, когда $p<0$, т. е. векторы $\mathbf{E}_{z}$ и $\mathbf{v}_{z}$ противоположны по направлению и $\partial \gamma / \partial z>0$. При $p>0$ (векторы $\mathbf{E}_{z}$ и $\mathbf{v}_{z}$ совпадают по направлению и $\partial \gamma / \partial z<0)$ параметр $a<0$, и первое слагаемое в $(15)$ работает на усиление неустойчивости.

Введем безразмерную амплитуду отклонения оси пучка $Y_{1}=Y / R_{b}$ и продольную координату $z_{1}=z k_{\beta 0}$, где

$$
k_{\beta 0}=\sqrt{\frac{I_{b}}{I_{A 0}}} \frac{1}{R_{b}}
$$

- бетатронное волновое число, расчитанное для начального значения энергии пучка. Здесь $I_{b}-$ полный ток пучка, $I_{A 0}$ - предельный ток Альфвена, рассчитанный для начального значения лоренц-фактора $\gamma_{\theta}, R_{b}-$ характерный радиус пучка.

Тогда уравнение (15) принимает вид

$$
\frac{\partial^{2} Y_{1}}{\partial z_{1}^{2}}=-\frac{a}{k_{\beta 0}} \frac{\partial Y_{1}}{\partial z_{1}}-\frac{2 f_{n} G\left(Y_{1}, \eta\right)}{\pi} .
$$

Тогда сравним по модулю вклад первого и второго слагаемых в правой части уравнения (17)

$$
T=\frac{2 f_{n} k_{\beta 0} G\left(Y_{1}, \eta\right)}{\pi|a|\left|\partial Y_{1} / \partial z_{1}\right|} .
$$



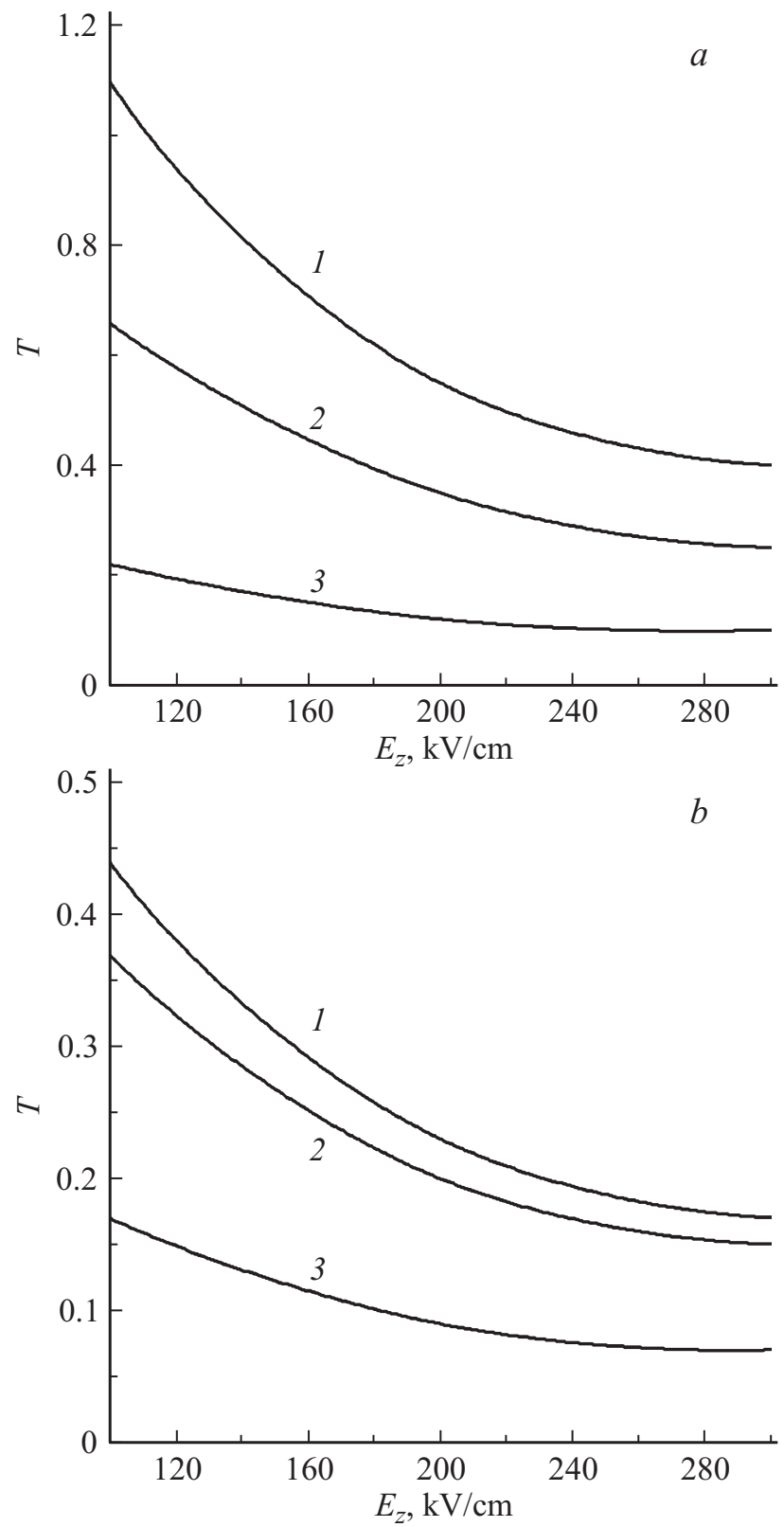

$a$ - зависимость величины $T$ от $E_{z}$ при следующих параметрах задачи: $\eta=R_{b} / R_{c h}=1, R_{b}=0.5 \mathrm{~cm}, \beta \sim 1, I_{b}=1 \mathrm{kA}$, $f_{n}=0.5, Y_{1}=Y_{0} \exp \left(b z_{1}\right), b=0.2$ : кривая 1 соответствует бизразмерной амплитуде поперечного отклонения оси симметрии пучка от оси ионного канала $Y_{1}=1$, кривая $2-Y_{1}=2$, $3-Y_{1}=4 . \quad b-$ полагается $\eta=R_{b} / R_{c h}=0.5$. нумерация кривых аналогична нумерации рисунка, $a$.

На рисунке представлена зависимость величины $T$ от $E_{z}$ при следующих параметрах задачи: $\eta=R_{b} / R_{c h}=1$, $R_{b}=0.5 \mathrm{~cm}, \beta \sim 1, I_{b}=1 \mathrm{kA}, f_{n}=0.5, Y_{1}=Y_{0} \exp \left(b z_{1}\right)$, $b=0.2$. Например, при $Y_{0}=0.02 R_{b}$ и $b=0.2$ случай $Y_{1}=1$ достигается при $z_{1}=19.56$, случай $Y_{1}=2-$ при $z_{1}=23.03$, и, наконец, третий случай - при $z_{1}=26.5$.

На рисунке $b$ представлена зависимость величины $T$ от $E_{z}$ при тех же параметрах задачи, что на рисунке $a$, но при $\eta=R_{b} / R_{c h}=0.5$.
Из рисунков следует, что трекинг-сила (при $a>0$ ) или детрекинг-сила (при $a<0)$, обусловленные влиянием внешнего продольного электрического поля $E_{z}$ на динамику РЭП в режиме ИФ, при больших амплитудах отклонения оси пучка $Y_{1}$ сильно зависят от величины $E_{z}$ и отношения характерных радиусов пучка и ионного канала, и могут по величине превышать силу пучковоканального взаимодействия.

\section{Заключение}

Получена формула для расчета силы, вызванной торможением (или ускорением) РЭП, распространяющегося вдоль ионного плазменного канала в режиме ионной фокусировки при наличии внешнего продольного электрического поля. Проведено сравнение по величине указанной силы и силы, обусловленной электрическим взаимодействием пучка и ионного канала при разных значениях напряженности внешнего электрического поля и амплитуды отклонения РЭП в поперечном направлении относительно оси канала. Показано, что при торможении пучка во внешем электрическом поле, рассматриваемая сила работает на уход пучка из канала (детрекингэффект), а при ускорении РЭП в указанном поле данная сила становится возвращающей (трекинг-эффект). Кроме того, показано, что сужение канала, когда отношение характерного радиуса пучка к характерному радиусу канала увеличивается, приводит к уменьшению исследуемой силы относительно силы электрического взаимодействия системы пучок-канал. При всех рассмотренных при численном расчете напряженностях внешнего электрического поля величина исследуемого параметра $T$ почти всюду меньше единицы. Это говорит о том, что сила пучково-канального взаимодействия превышает по величине силу, вызванную торможением (или ускорением) пучка. Однако данные силы соизмеримы и учет указанной силы может оказывать заметное влияние на динамику развития ионной шланговой неустойчивости.

\section{Список литературы}

[1] Рухадзе А.А., Богданкевич Л.С., Росинский С.Е., Рухлин B.Г. Физика сильноточных релятивистских электронных пучков. М.: Атомиздат, 1980. 167 с.

[2] Миллер $Р$. Введение в физику сильноточных пучков заряженных частиц. М.: Мир, 1984. 432 с.

[3] Колесников Е.К., Мануйлов А.С., Филиппов Б.В. Динамика пучков заряженных частиц в газоплазменных средах. СПб.: Изд-во СПбГУ, 2002. 98 с.

[4] Колесников Е.К., Мануйлов А.С., Зеленский А.Г. Динамика релятивистских электронных пучков в режиме ионной фокусировки. Воскресенск.: Изд-во Позитив, 2013. 104 с.

[5] Buchanan H.L. // Phys. Fluid. 1987. Vol. 30. N 1. P. 221-231.

[6] Колесников Е.К., Мануйлов А.С. // ЖТФ. 2000. Т. 70. Вып. 5. C. $68-73$. 